\title{
AutoCAD2010 based automatic dimensioning of size and tolerance
}

\author{
Bao Jiading ${ }^{1, \mathrm{a}}$, Zhao Rui ${ }^{2, \mathrm{~b}}$, Sun Yonghou ${ }^{1, \mathrm{c}}$, Liu fuyun ${ }^{1, \mathrm{~d}}$,Huang Meifa ${ }^{1, \mathrm{e}}$ \\ ${ }^{1}$ Guilin University of Electronic Technology, Guilin 541004, China \\ ${ }^{2}$ Lijing College of Guangxi Normal University, Guilin 541006, China \\ a jdbao@guet.edu.cn, ${ }^{b} 328886517 @ q q . c o m,{ }^{c}$ syh@guet.edu.cn, dliufuyun310@aliyun.com, ,hmhm \\ f@guet.edu.cn
}

Keywords: Dimensional chain technology; CAT; AutoCAD; Secondary development; ObjectARX

\begin{abstract}
Computer Aided Tolerancing (CAT) is the key technology of CAD and CAM integration. However, nowadays the tolerance analysis and synthesis in CAD/CAM are mostly realized by manual methods or the experience of the designers. Thus this not only increased the workload of the designers but also wasted a lot of time. As a result, this paper applies dimensional chain technology in AutoCAD2010 software through ObjectARX development tool to realize the automatic dimensioning and tolerancing. In the process of software secondary development, the deep first search method is adopted to generate dimensional chain automatically, and worst-case method and minimum cost method are separately applied to carry out tolerance analysis and synthesis. Finally an example is presented to validate the given method.
\end{abstract}

\section{Introduction}

With the rapid development of computer science and advanced manufacturing technology, traditional manufacturing has changed radically. Recently significant breakthrough has been made in CAD, CAM, and CAPP, and the integration of CAD/CAM/CAPP is the development trend in modern manufacturing ${ }^{[1]}$. CAT is one of the key technologies in CAD/CAM integration and many research works have been carried out. Dimensional chain technology is the key in CAT. In the present CAD software system, nominal geometry information (ideal information) can be well expressed, but variational geometry information (tolerance information) is just in the form of text that cannot be applied to tolerance analysis and synthesis ${ }^{[2]}$. Whereas tolerance analysis and synthesis can be achieved by using extremum method, probabilistic method, Monte Carlo method or minimum cost method based on dimensional chain technology.

This paper aims at applying dimensional chain technology in AutoCAD2010 to realize the automatic dimensioning and tolerancing. By this, designers can specify tolerances easily and accurately in accordant with the new generation geometrical product specifications and verifications (GPS) standards. Finally, a classical example is presented to validate the rationality and effectiveness of the given method.

\section{Generation of dimensional chain}

\subsection{Dimensional chain}

Dimensional chain is a closed size group in a certain order in designing and machining of the parts $^{[3]}$. Linear dimensional chain and flat dimensional chain are commonly seen in practical production. Flat dimensional chain is always changed into linear dimensional chain using projection method. Among all the dimensions in dimensional chain, the dimension which indirectly guaranteed is called critical dimension, and the others are referred to functional dimensions. Functional dimensions can be classified as increasing links and decreasing links according to their influence properties to the critical link. We can judge the increasing links and decreasing links by following methods. Choosing any one direction (Clockwise or counterclockwise) along the dimensional chain. If the direction of the functional dimension and the critical dimension are the opposite, we define the 
functional dimension as increasing link and, otherwise, we name it as decreasing link ${ }^{[4]}$. As shown in Fig.1, if we assume $A_{\Sigma}$ as critical dimension, then $A_{2}$ and $A_{3}$ are called increasing link as their directions are opposite with the critical link $A_{\Sigma}$, and $A_{1}$ is called decreasing link as its direction is the same with critical link $A_{\Sigma}$.

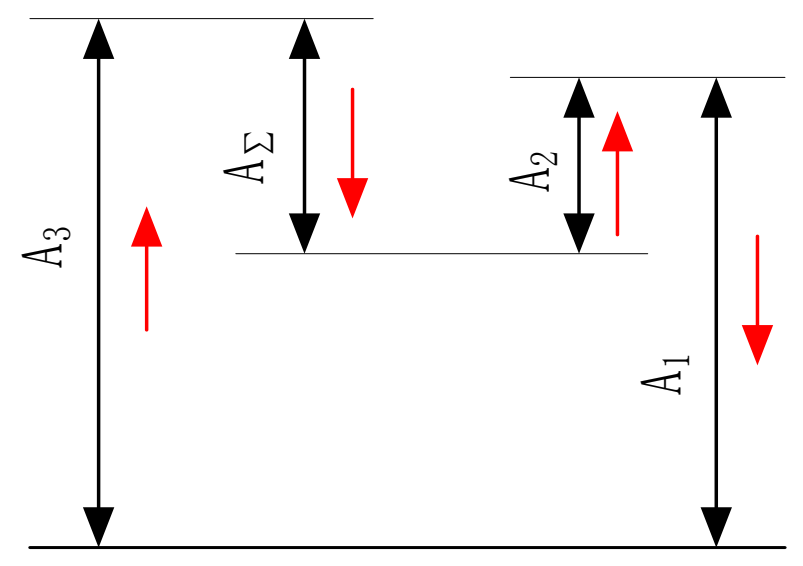

Fig.1. Dimensional chain

The deep first search method is adopted to generate dimensional chain automatically in this paper. This method is a constant recursive search process which seeks for associated elements. In the generation of dimensional chain, we specify the two sides of the critical dimension as its two key elements. Choosing one element as the starting element, and then search its associated element. The ending element of some dimensions is also the starting element of another dimension. All the dimensional chain will be found through this associated relationship. If all the associated dimensions elements are visited in the database, the search of dimensional chain is over.

\subsection{Calculations in dimensional chain}

\subsubsection{Tolerance analysis and synthesis}

Calculation in dimensional chain can be divided into tolerance analysis and synthesis to solve the problems of fundamental dimension and the tolerance between critical dimensions and functional dimensions.

Tolerance analysis can be used to solve the dimension, tolerance and deviation of the critical dimension if all the dimensions and tolerances of the functional dimensions are known. Tolerance analysis is always applied in the precision check.

Tolerance synthesis is used to solve the dimensions, tolerances and deviations of the functional dimensions based on the known critical dimensions and tolerances. Tolerance synthesis is always applied in the tolerance design. Tolerance synthesis is the inverse process of tolerance analysis ${ }^{[5]}$.

In the design process of the parts, tolerance analysis is firstly conducted when the dimensions, tolerances and deviations of all the functional dimensions are known. Then the dimension, tolerance and deviation of the critical dimension will be acquired. If the dimension, tolerance and deviation of the critical dimension do not meet the design requirement, then the tolerance synthesis should be redesigned.

In the design process of the parts, if the dimensions, tolerances and deviations of all the functional dimensions are not completely determined, the tolerance synthesis can be directly conducted. The results of the tolerance synthesis can be treated as the final values, and they can also be regarded as the reference values.

\subsubsection{Calculation methods}

The calculation methods in dimensional chain mainly including worst-case method, probabilistic method, Monte Carlo method, and minimum cost method.

As the worst-case method is the simplest method which can completely ensure the interchangeability of the parts, this paper mainly adopts this method to carry out tolerance analysis.inimum cost method can get the best economic benefits, which determines functional 
tolerance based on the functional relationship between cost and tolerance. So this paper primarily applies this method to carry out tolerance synthesis ${ }^{[6-7]}$.

The following example shown in chapter 3.4 is mainly introduced by worst-case method. Hence we just introduce minimum cost method here.

Negative square model is adopted by analyzing and fitting a large amount of data in the actual production. This model can simply and truly reflect the relationship between tolerance and cost. The expression of negative square model is shown as formula (1).

$$
c(t)=a+\frac{b}{t^{2}}
$$

where $t$ stands for dimensional tolerance, and $c(t)$ stands for machining cost. $a$ is a constant which independent on the tolerance, while $b$ is also a constant that related to the tolerance. In tolerance synthesis, the objective function of optimal tolerance allocation can ensure the minimum cost of total manufacturing, shown as formula (2).

$$
\min \left(c_{\Sigma}\right)=\min \left[\sum_{i=1}^{l} a_{i}+\frac{b_{i}}{t_{i}{ }^{2}}\right]
$$

where $l$ is the number of unknown functional dimension. The constraint conditions are the equality relationships between critical dimension and functional dimension. Then the Lagrange multiplier method is used to conduct a first order partial derivative for each unknown parameter. Combine all the equations and set the derivative value as zero, then tolerance value of unknown functional dimension will be obtained.

\section{Application of dimensional chain}

\subsection{Dimensioning and tolerancing}

In AutoCAD, ObjectARX development tool is applied to establish an additional function menu named automatic dimensioning and tolerancing menu. The steps of automatic dimensioning and tolerancingare shown as follows.

Step 1: the drawing of the parts. According to the design plan of the designers, the drawing of the parts are completed in AutoCAD software.

Step 2: the automatic generation of the dimensional chain. The automatic algorithm of the dimensional chain is an iterative process. Under the premise of full constraints to the parts, all the component chains must be found to generate the proper dimensional chain through the repeated inquires and iterations. Click the critical dimension button to pick the critical dimension in the part drawing, as shown in Fig. 2. Next, click dimensional chain search button to select parts of faces from left to right or from up to down. As a result, the dimensional chain is automatically searched out.

Step 3: the calculation of the dimensional chain. According to the situation of the part drawing, choose tolerance analysis or tolerance synthesis, as shown in Fig. 2. Then click functional dimension marking information button to extract and save the information of functional dimensions. Finally, press the result display button to finish the tolerance design and display the results in the List Control. The final effect is used to complete the automatic dimensioning andtolerancing. 


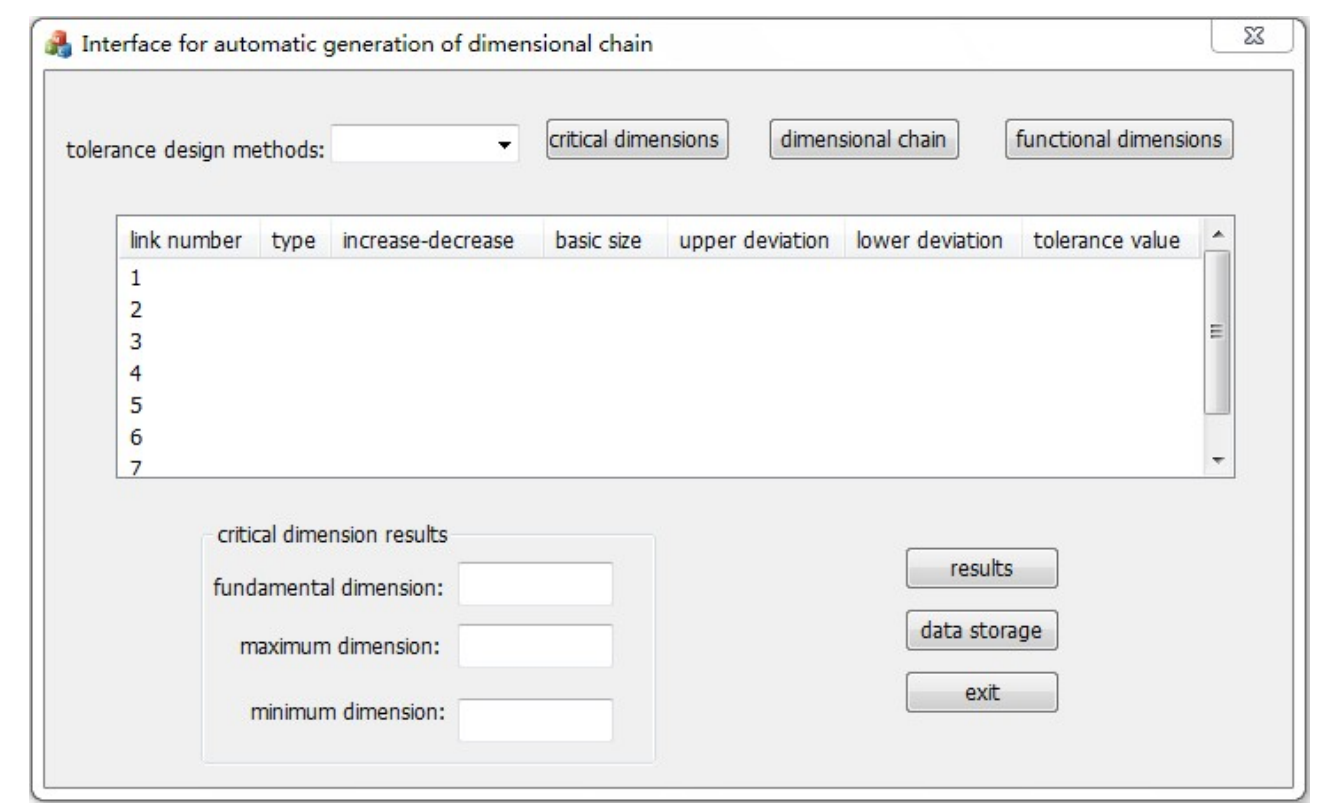

Fig.2. Interface for automatic generation of dimensional chain

\subsection{Establishment of dimensional chain model}

The expression and processing of dimensional chain in computer are the basis of tolerance analysis and synthesis. The dimensional chain model established in this paper is shown as follows:

Class DIMCHAIN \{

Int DimID; 'dimension ID

double dbasesize; 'fundamental dimension

double dTol; 'tolerance value

double dES; $\quad$ 'upper deviation

double $\mathrm{dEI}$ 'lower deviation

double dTranCoe 'transfer coefficient

\} ;

This representation structure is suitable to linear and plane tolerance chain. And whether the dimension is increasing link or decreasing link can also be determined by transfer coefficient.

\subsection{Secondary development}

AutoCAD is one of the most important CAD softwares. It is a crucial tool to exchange design ideas for project planners. Besides, the Open Architecture of AutoCAD makes it easier for secondary development ${ }^{[8]}$. Secondary development technology is always utilized to develop the customized, professional and integrated software which meets the actual needs.

The system introduced in this paper is mainly implemented on the development platform of AutoCAD2010. And ObjectARX2010 is used as the development tool. As the latest generation development tool of AutoCAD software, ObjectARX can share the address space of the AutoCAD, access to the AutoCAD database and establish the new commands with the same operating mode of AutoCAD itself ${ }^{[9]}$. This paper makes use of Visual studio2008.net and MFC to design and develop an object-oriented CAD/CAT integration system.

\subsection{Example for application}

An example is presented to show the process of the dimensional chain calculation using dimensional chain technology, as shown in figure 3. The critical dimension should be limited to $A_{0}=12_{-0.1}^{+0.1}$.

Firstly, we choose $A_{0}$ as the critical dimension in AutoCAD software. Click OK button, and then the related dimensional chain will be generated and seen in AutoCAD software. Secondly, decide tolerance analysis or tolerance synthesis according to the actual situation. It seems apparent that we should make tolerance synthesis solve this example. The final results are achieved based on the extremum method. The calculative idea of the extremum method is expressed as follows:

(1) Check the fundamental dimension $\mathrm{A}_{0}$ of the critical dimension 
$A_{0}=A_{1}+A_{2}-A_{3}=22+27-37=12$

(2) Check the tolerance of the critical dimension

$T_{0}=T_{1}+T_{2}+T_{3}=0.05+0.02+0.03=0.10$

(3) Check the upper deviation and lower deviation of the critical dimension

$E S_{0}=E S_{1}+E S_{2}-E I_{3}=0.03+0-(0.03)=0.06$

$E I_{0}=E I_{1}+E I_{2}-E S_{3}=-0.02-0.02-0=-0.04$

Where ES is the upper deviation and EI is the lower deviation.

So the full expression of the critical dimension $A_{0}$ is $A_{0}=12_{-0.04}^{+0.06}$. The critical dimension $A_{0}$ is within the scope of the given requirement $A_{0}=12_{-0.1}^{+0.1}$. As a result, we can conclude that all the dimensions and tolerances meet the design requirements.
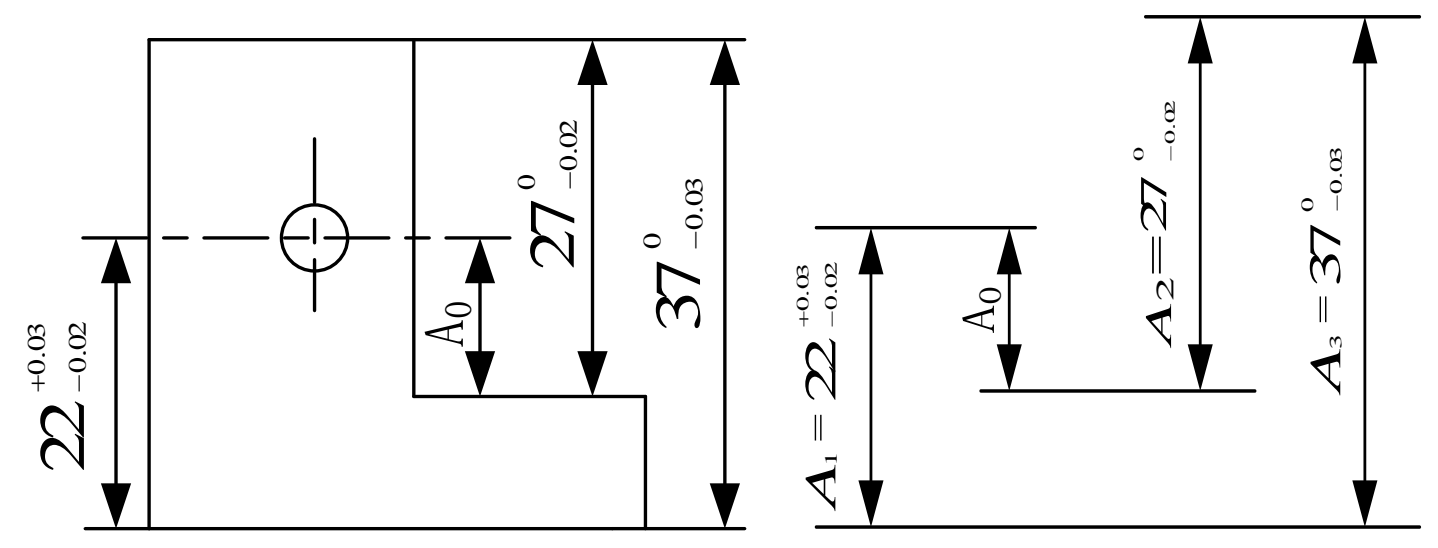

Fig.3. Example for the calculation of the dimensional chain

\section{Conclusions}

This paper has discussed the dimensional chain technology in detail and has applied it in AutoCAD2010 through secondary development technology. As a result, the automatic dimensioning and tolerancingare completed successfully. An example is presented to validate the rationality and effectiveness of the given method. The example shows that the dimensional chain technology is an important means of ensuring the product quality, simplifying the processing and reducing the cost.

\section{Acknowledgements}

This paper was supported by Natural Science Foundation of China (No. 51265006 and No. 51365009), Guangxi University of science and technology research projects(NO.KY2015YB102), the Guangxi Key Laboratory of Manufacturing Systems and Advanced Manufacturing Technology (No. 13-051-09-009Z and No. 14-045-15-008Z).

\section{References}

[1] Xiao Di, Zhang Genbao. The research of mechanical assembly modeling \& automatic dimensioning technique on parts [D]. Chongqing: Chongqing University Press, 2002.

[2] Shah, J.J. Dimension and tolerance modeling and transformations in feature based design and manufacturing. Journal of Intelligent Manufacturing, 1998,475-488.

[3] D.Dedoncker, A.Spencer. Assembly tolerance analysis with simulation and techniques [c]. SAE Transactions, 1987, 96(1):1061-1067. 
[4] Zhang Rongrui. The theory and application of the dimensional chain. Beijing: Mechanical Industry Press, 1986.

[5] Liu Jianye, Yang Dongbai. Research and implementation of computer aided tolerancing based on CAD platform [D]. Beijing: Academy of Machinery Science \& Technology, 2003.

[6] Department of Mathematics and Mechanics of Zhong Shan University, Theory of probability and mathematical Statistics, vols. 1 and 2.Higher Education Press, 1980, pp. 148-158; pp. 343-344; pp. 363.

[7] Z. Jianzhong, Monte-Carlo method practice and understanding of mathematics, first issue, 1974, pp. 28-33.

[8] Wei Yongle, Liu Mingxin, Su Meng. Research of secondary development on AutoCAD based on ObjectARX [J]. Scientific Journal of Machinery, 2005(32).

[9] Yu Xiaorong, Guo Changyan, Chen Gang. The research of AutoCAD secondary development framework combined Objectarx and C\#[J]. Science Technology and Engineering, 2010 , 10(20):5085-5090. 